BMJ Open

Ophthalmology

\title{
Anxiety and depression in inflammatory eye disease: exploring the potential impact of topical treatment frequency as a putative psychometric item
}

\author{
Georgios Vakros (D) , ,2 Paolo Scollo, ${ }^{1,2}$ James Hodson, ${ }^{3}$ Philip I Murray (D) ,,2 \\ Saaeha Rauz ${ }^{1,2}$
}

To cite: Vakros G, Scollo P, Hodson J, et al. Anxiety and depression in inflammatory eye disease: exploring the potential impact of topical treatment frequency as a putative psychometric item. BMJ Open Ophthalmology 2021;6:e000649. doi:10.1136/ bmjophth-2020-000649

GV and PS contributed equally.

Received 29 0ctober 2020 Revised 7 January 2021 Accepted 21 January 2021
D Check for updates

C Author(s) (or their employer(s)) 2021. Re-use permitted under CC BY-NC. No commercial re-use. See rights and permissions. Published by BMJ.

${ }^{1}$ Academic Unit of Ophthalmology, Institute of Inflammation and Ageing, University of Birmingham, Birmingham, UK

${ }^{2}$ Birmingham and Midland Eye Centre, Sandwell and West Birmingham Hospitals NHS Trust, Birmingham, UK ${ }^{3}$ Department of Biostatistics, Institute of Translational Medicine, University Hospitals Birmingham NHS Foundation Trust, Birmingham, UK

Correspondence to Miss Saaeha Rauz; s.rauz@ bham.ac.uk

\section{ABSTRACT}

Objective To evaluate whether topical therapy is linked to scores related to anxiety, depression and quality of life (QoL) in inflammatory eye disease (IED).

Methods and analysis Patients with ocular surface disease (OSD, $\mathrm{N}=100)$ and Uveitis $(\mathrm{N}=100)$ completed self-administered validated questions on ocular symptoms and well-being, with supplemental questions on eye drop frequency.

Results Forty (20\%) patients had scores consistent with depression and 33 (17\%) anxiety. Anxiety, depression, QoL and OSD index (OSDI) scores did not differ significantly between OSD and Uveitis groups. In those with anxiety or depression, QoL was significantly reduced in all WHO Quality Of Life-BREF domains (all $p<0.001$ ). Multivariable analysis considering demographic and diseaserelated factors found daily topical drop frequency to be independently associated with anxiety $(p=0.009)$ but not depression $(p=0.300)$.

Conclusion A high proportion of patients with IED demonstrated scores indicative of anxiety and depression. Preliminary evidence suggests that the frequency of topical eye drops potentially plays a significant role in the psychological health status of patients with IED .

\section{INTRODUCTION}

Depressive disorders share the common feature of a 'sad, empty or irritable mood', associated with somatic and cognitive alterations that significantly impair an individual's capacity to function, while anxiety disorders are behaviours characterised by excessive fear and anxiety, including hypervigilance and cautious or avoidance behaviours, secondary to an abnormal anticipation of future threat. ${ }^{1}$ Anxiety and depressive disorders frequently coexist are often not easily differentiated from one another and can have a chronically disabling impact on physical well-being and quality of life (QoL). ${ }^{2}$ Recent national survey data from England revealed that the prevalence of the two most common mental disorders, generalised anxiety disorder and depression, has increased in recent years,

\section{Key messages}

What is already known about this subject?
Published data demonstrate that anxiety and de-
pression is high in IED sufferers and that dry eye
disease is associated with depression.
What are the new findings?
This is the first study using real-world data to assess
the effect that eye drop frequency might have on pa-
tient well-being and demonstrates that topical med-
ication is associated with an increased likelihood of
suffering from anxiety.
How might these results change the focus of
research or clinical practice?
The ocular surface disease index patient reported
outcome scoring system may have a place in mea-
suring patient experience in living with non dry-eye
inflammatory eye disease states such as uveitis.
Patient and public involvement and engagement is
essential e in providing feedback on the design of a
study and guides towards more patient-centric, real-
world and public accessible studies.

reaching $5.9 \%$ and $3.3 \%$, respectively. These rates were found to be higher in women and in those of working age (16-64 years), compared with those over 65 years. ${ }^{3}$

Both anxiety and depression are more prevalent in the presence of chronic medical illnesses. ${ }^{45}$ With an overlay of visual disability, this is also true in context of chronic inflammatory eye diseases (IED), as many patients require repeated visits to an ophthalmologist, often needing lifelong treatment, including frequently applied topical therapy that can impact on daily activities, general well-being and QoL.

Evidence suggests that anxiety and depression are frequently underdiagnosed comorbidities that significantly impact on health-related QoL. ${ }^{6-12}$ The cause for increased rates of anxiety and depression 
in patients with IED specifically is multifactorial. Poor visual function has been identified as a significant risk factor for depression and reduced QoL in patients with ocular surface disease (OSD) ${ }^{713}$ and Uveitis. ${ }^{14-16}$ Unresolved pain is also likely to play an important role in the development of mental illness in many chronic medical conditions. ${ }^{17}$ Recent studies confirm that neuropathic ocular pain specifically is not only prevalent in those with dry eye disease (DED) but also correlates with DED severity and persistence. ${ }^{18-22}$ From a patient perspective, a lack of understanding of their condition may inhibit treatment adherence, ${ }^{23}$ perpetuating both physical and mental morbidities. ${ }^{5}$ A lack of understanding of IED on the behalf of a patient's social contacts may also have an additional impact. Gender has also been found to be of importance in mental morbidity, ${ }^{24}$ as has workplace dissatisfaction..$^{25}$

Unrecognised mental illness in chronic IED is a serious clinical concern for patients, in terms of their cognitive and physical functioning, and productivity. Poor health-related QoL may also impact on the medical management of IED, which could lead to issues with treatment adherence, further compounding morbidity. While the majority of patients with IED are managed with topical therapy, either alone or in combination with systemic therapy, we have frequently observed patient concerns regarding their arduous eye drop regimens. We wished to explore this further as, to our knowledge, no previous study has examined whether the frequency of topical treatment application requested by medical professionals could contribute to anxiety, depression and poor QoL using real-world data. Furthermore, no study has compared OSD and Uveitis patient groups, both important sight-threatening inflammatory conditions that routinely require frequent daily eye drops.

\section{MATERIAL AND METHODS}

\section{Study population}

The study recruited 100 consecutive patients with OSD (clinician: SR) and 100 consecutive patients with Uveitis (clinician: PIM) seen in the IED Service at the Birmingham \& Midland Eye Centre, Birmingham, UK over a 4-month period (August to December). Inclusion criteria were the ability and willingness to consent to the study and to read and answer questions in English without assistance (in order to maintain anonymity). Additionally, patients were only eligible for inclusion in the study if they were on a stable treatment regime, with their current eye drop frequency being unchanged for at least 4 weeks; this was assessed by a screening question.

OSD comprises a group of disorders, of diverse pathogenesis, in which disease results from the failure of mechanisms responsible for maintaining a healthy ocular surface. Patients included in this study had mucous membrane pemphigoid, Stevens-Johnson syndrome/toxic epidermal necrolysis, graft-versus-host disease, atopic/vernal keratoconjunctivitis or primary/ secondary Sjögren's syndrome. Management of the associated OSD involves control of blepharitis, dry eye, filamentary keratitis, keratinisation, lid malposition and persistent epithelial defect as well as the identification and avoidance of toxicity. Inflammation associated with the underlying disorder demands the use of systemic immunosuppressive therapy. Topical therapy involves nonpreserved lubricating eye drops at varying frequencies dependent on the patient response (6x daily to every $15 \mathrm{~min}$ ) and/or ointments, which may be combined with a topical corticosteroids or calcineurin inhibitors. For refractory cases, serum eye drops provide nutritive substitutes.

Uveitis comprises a group of conditions characterised by intraocular inflammation and can be classified anatomically into anterior, intermediate, posterior or panuveitis. Uveitis may be infectious or noninfectious and, if the latter, may be part of an associated systemic disease, such as the HLA-B27 group of diseases and sarcoidosis. Patients with anterior segment inflammation are managed with topical corticosteroids, with the frequency dictated by disease activity and response to treatment. Some patients may also require the use of a topical cycloplegic. It is not uncommon for patients with uveitis to also need lubricating eye drops on an as-required basis.

\section{Patient and public involvement}

The West Midlands Patient Involvement Group in Uveitis was involved in the evaluation of the OSD index (OSDI) score prior to the conception of this study, and they agreed that the OSDI also reflected some of the chronic uveitis symptomatology they had experienced. In addition, the OSD patient and public involvement (PPI) highlighted that the commitment required by the patients with regards to treatment is not reflected in the OSDI or Hospital Anxiety and Depression Scale (HADS). As such, both groups recommended two additional questions, which were added at the end of the HADS and are detailed below. Patients and/or the public were not involved in the conduct or reporting of the study or the dissemination plans of this research.

\section{Study design and procedures}

Participants completed an anonymous, self-administered questionnaire, comprised of demographic data (decade of age, gender, ethnicity, geographical area, education and working status), treatment data (current use and frequency of topical and/or systemic medications) and the OSDI. In addition, validated well-being questionnaires, including the HADS and WHO Quality of life-BREF (WHOQOL-BREF) were completed. Participants completed the questionnaires while in the clinic waiting area prior to their medical consultation, and returned the completed forms to a generic questionnaire return box located in the waiting room, to retain patient anonymity.

The OSDI is a validated 12-item questionnaire that assesses patients' DED symptom severity ${ }^{26}$ and is recommended by DEWS II as a diagnostic screening 
instrument. ${ }^{21}$ It is divided into three main categories, with specific questions on: experience of symptoms (light sensitivity, grittiness, ocular pain, blurred vision, poor vision); effect on performing daily tasks (reading, night driving, working with electronic devices, watching television) and discomfort in specific environments (windy conditions, low humidity areas, air-conditioned areas). Each question has six potential responses on a scale ranging from 0 to 5 (none of the time, some of the time, half of the time, most of the time, all of the time) or 'not applicable/not answered'. The OSDI score is calculated as the mean of the responses of all of the questions answered by the respondent, which is then multiplied by 25 , to give a score in the range $0-100$, with higher scores representing more severe symptomatology. Based on the resulting scores, patients can be classified as having normal (OSDI $=0-12)$, mild (13-22), moderate (23-32) or severe (33+) symptomatology. As mentioned above, while the OSDI was intended for use in OSD, a patient involvement group indicated that many of the questions were applicable to uveitis because of the similarity of presenting symptoms and how these affect daily activities. In addition, newer insights into DED as an inflammatory condition indicate that the OSDI also has a role in uveitis. ${ }^{27}$ On this basis, the OSDI questionnaire was used for both groups.

The HADS is a validated 14-item self-administered screening questionnaire that determines the levels of anxiety and depression that a physical health sufferer experiences. ${ }^{28}$ It has seven items related to anxiety and seven relating to depression, based on patient feelings in the last week. Each question has four choices, with scores ranging from 0 to 3 (not at all, from time to time, a lot of the time, most of the time). These are then added together, to give scores ranging from 0 to 21 for anxiety and depression. Large systematic reviews have identified 'cut-off points', with a score of less than or equal to 7, indicating a 'normal' response, scores ranging from 8 to 10 being classified as 'borderline' and scores of 11 or greater constituting a 'case'. ${ }^{29}$ A large literature review assessing the validity of the HADS identified 747 papers using the instrument and found the HADS to perform well in its assessment of anxiety and depression 'caseness' and severity, with a sensitivity and specificity of approximately 0.80 for both the HADS-A (anxiety) and HADS-D (depression) components. ${ }^{30}$

Finally, the PPI recommended additional questions, which were appended to the end of HADS and related to whether eye drops affect mood and whether they improve symptoms. Patients were asked 'Do you become low about the number of eye drops' and 'Do your eye drops improve your symptoms', both with the four response options: not at all, not much, sometimes, always.

The WHOQOL-BREF is a modified, shorter version of the WHOQOL-100 questionnaire, using 26 items to assess four broad domains: physical health, psychological health, social relationships and environment, to give a QoL profile for each participant tested, based on patient feelings in the past 4 weeks. ${ }^{31}$ Each of the items has five choices that are scored on a scale from 1 to 5 . The items are then combined to form total scores for the four domains. In each case, the mean of all of the items within a domain is calculated, before being multiplied by 4 , in order to give a score in the range 4-20, which is comparable with the score of the WHOQOL-100 questionnaire. These scores are scaled in a positive direction, whereby higher values relate to higher QoL.

\section{Statistical analysis}

Initially, a range of demographics, treatment and QoLrelated factors were compared between the OSD and Uveitis groups. Comparisons of nominal values were performed using Fisher's exact test, with Mann-Whitney $\mathrm{U}$ test used for ordinal and continuous variables. Associations between the QoL scores and treatment factors were performed using a similar approach. Correlations between ordinal variables were assessed using Kendall's $\tau$, while comparisons across nominal factors with more than two groups were performed using Kruskal-Wallis tests, followed by post hoc Dunn's tests, where overall significance was detected.

A multivariable analysis was then performed, to assess whether eye drop frequency was independently associated with the depression and anxiety scores. These were treated as continuous variables in the analysis; however, since both scores followed skewed distributions, values were $\log _{10}$-transformed, after adding one to remove zeros, which had the effect of normalising the distribution and improving model fit. The resulting variables were then set as the dependent variables in general linear models, using a backwards stepwise approach for variable selection (with removal at $\mathrm{p}>0.1$ ). Where the eye drop frequency was not selected for inclusion in the final model, the variable was added into a new model alongside the factors identified as significant. The coefficients from the resulting models were then antilogged, such that they represented the estimated percentage increase in the depression/anxiety score per unit increase in continuous variables or the estimated percentage difference relative to the reference category for nominal variables.

All analyses were performed using IBM SPSS V.22 (IBM, Armonk, New York). Ordinal and continuous data are reported as medians with IQRs. Cases with missing data were excluded on a per-analysis basis. For all tests, the critical $p$ value for statistical significance was set at $\mathrm{p}<0.05$.

\section{Ethical approval}

Ethics approval was provided by 'Inflammation in Ocular Surface Disease' (LREC ref: 08/H1206/165; Birmingham East, North and Solihull Research Ethics Committee).

\section{RESULTS}

Table 1 shows the patient demographics for the whole cohort as well as for the OSD and Uveitis groups separately. 
Table 1 Inflammatory eye disease patient demographics and comparisons between OSD and Uveitis groups

\begin{tabular}{|c|c|c|c|c|}
\hline & All $(\mathrm{N}=200)$ & OSD $(N=100)$ & Uveitis $(\mathrm{N}=100)$ & $P$ value \\
\hline Age (years) ${ }^{*}$ & $54(41-68)$ & $58(43-71)$ & $51(39-63)$ & 0.062 \\
\hline Gender & & & & 0.057 \\
\hline Male & 74 & 44 & 30 & \\
\hline Female & 126 & 56 & 70 & \\
\hline Ethnicity† & & & & 0.172 \\
\hline White & 132 & 71 & 61 & \\
\hline Black & 18 & 7 & 11 & \\
\hline South Asian & 45 & 18 & 27 & \\
\hline Mixed & 5 & 4 & 1 & \\
\hline Education & & & & 0.853 \\
\hline Nil/technical skills & 66 & 35 & 31 & \\
\hline GCSE/A levels & 92 & 45 & 47 & \\
\hline University & 42 & 20 & 22 & \\
\hline Employment & & & & 0.117 \\
\hline Full/part/self & 77 & 32 & 45 & \\
\hline Student/unemployed & 31 & 16 & 15 & \\
\hline House-wife/husband & 23 & 10 & 13 & \\
\hline Retired & 69 & 42 & 27 & \\
\hline Oral corticosteroid & & & & 0.124 \\
\hline No & 156 & 83 & 73 & \\
\hline Yes & 44 & 17 & 27 & \\
\hline Eye drops per day & & & & $<0.001 \ddagger$ \\
\hline None & 28 & 9 & 19 & \\
\hline As required & 17 & 7 & 10 & \\
\hline 1-4 times & 88 & 32 & 56 & \\
\hline 5-7 times & 30 & 21 & 9 & \\
\hline Up to 2 hourly & 37 & 31 & 6 & \\
\hline
\end{tabular}

$P$ values are comparing the OSD and Uveitis groups and are from Fisher's exact tests, unless stated otherwise. Bold $p$ values are significant at $\mathrm{p}<0.05$.

${ }^{*}$ Age was reported to the nearest decade, hence medians and interquartile ranges are estimated assuming an even distribution of ages within each decade. The comparison between groups treated the decade of age as an ordinal variable, and was performed using a MannWhitney U test.

†UK Census 2011 Classification for ethnicities.

$\ddagger P$-value from a Mann-Whitney $U$ test, as the factor is ordinal.

A Level, Advanced Level; GCSE, General Certificate of Secondary Education; OSD, ocular surface disease.

The median age for the cohort as a whole was 54 years (IQR: 41-68), with the majority of patients being women $(\mathrm{N}=126,63 \%)$. The cohort was predominantly of white ethnicity $(66 \%)$, with the majority of patients educated to GCSE/A-level (46\%) or university (21\%) standard, and most patients either in employment $(39 \%)$ or retired $(35 \%)$. Comparisons between the OSD and Uveitis subgroups found no evidence of significant differences in any of the demographic factors considered. However, a significant difference in eye drop frequency was detected $(\mathrm{p}<0.001)$, with $52 \%$ of the OSD group requiring five or more drops per day, compared with $15 \%$ of those with uveitis.
Using the OSDI grading system, the majority $(51 \%)$ of patients reported significant symptoms (score: $33+$ ), with only $17 \%$ having an OSDI graded as normal (score: $0-12$ ). The OSDI score was not found to differ significantly between the OSD and Uveitis groups $(p=0.066)$, with median scores of 35 and 30, respectively. The QoL scores, as measured by the WHOQOL-BREF domains, were also similar in the OSD and Uveitis groups (table 2).

The HADS screening questionnaire classified $17 \%$ of the total cohort as having a score compatible with anxiety (score: $11+$ ), with a further $10 \%$ being in the borderline category (score: 8-10). Similar rates of depression were detected, with $20 \%$ of patients having a score compatible 
Table 2 Symptoms, depression, anxiety and QoL of patients in the OSD and Uveitis subgroups

\begin{tabular}{|c|c|c|c|c|}
\hline & Overall & OSD & Uveitis & $P$ value \\
\hline OSDI & & & & 0.066 \\
\hline Median (IQR) & $33(18-56)$ & $35(21-68)$ & $30(16-50)$ & \\
\hline$\%$ Normal (0-12) & $33(17 \%)$ & $17(18 \%)$ & $16(16 \%)$ & \\
\hline$\%$ Mild (13-22) & $28(14 \%)$ & $10(10 \%)$ & $18(19 \%)$ & \\
\hline \% Moderate (23-32) & 35 (18\%) & 15 (15\%) & $20(21 \%)$ & \\
\hline \% Severe (33+) & $98(51 \%)$ & $55(57 \%)$ & $43(44 \%)$ & \\
\hline Anxiety score & & & & 0.273 \\
\hline Median (IQR) & $4(2-8)$ & $5(3-8)$ & $4(2-8)$ & \\
\hline$\%$ No anxiety $(\leq 7)$ & $147(74 \%)$ & $74(74 \%)$ & $73(73 \%)$ & \\
\hline \% Borderline (8-10) & $20(10 \%)$ & $11(11 \%)$ & $9(9 \%)$ & \\
\hline \% Anxiety (11+) & $33(17 \%)$ & $15(15 \%)$ & $18(18 \%)$ & \\
\hline Depression score & & & & 0.458 \\
\hline Median (IQR) & $5(3-9)$ & $6(3-10)$ & $5(2-9)$ & \\
\hline$\%$ No depression $(\leq 7)$ & $132(66 \%)$ & $63(63 \%)$ & $69(69 \%)$ & \\
\hline$\%$ borderline (8-10) & $28(14 \%)$ & $16(16 \%)$ & $12(12 \%)$ & \\
\hline \% Depression (11+) & $40(20 \%)$ & $21(21 \%)$ & 19 (19\%) & \\
\hline \multicolumn{5}{|l|}{ WHOQOL-BREF domain } \\
\hline Physical & $14(12-16)$ & $14(11-16)$ & $14(12-16)$ & 0.651 \\
\hline Psychological & $15(13-17)$ & $15(13-17)$ & $15(13-17)$ & 0.414 \\
\hline Social relationships & $16(13-18)$ & $16(13-18)$ & $16(13-18)$ & 0.696 \\
\hline Environment & $16(14-18)$ & $16(14-18)$ & $16(13-17)$ & 0.295 \\
\hline
\end{tabular}

Data reported as N (\%) or as Median (IQR), as applicable, with p values from Mann-Whitney tests.

Data for the OSDI were only available in $\mathrm{N}=194$ ( $\mathrm{N}=97$ OSD and $\mathrm{N}=97$ Uveitis), and the WHO-BREF domains were recorded in $\mathrm{N}=95-97$ OSD and $\mathrm{N}=95-99$ Uveitis.

GCSE, General Certifate of Secondary Education; OSD, ocular surface disease; WHOQOL-BREF, World Health Organisation Quality Of Life Questionnaire.

with depression and $14 \%$ being borderline. Neither the anxiety $(\mathrm{p}=0.273)$ nor depression $(\mathrm{p}=0.458)$ scores were found to differ significantly between the OSD and Uveitis groups (table 2).

Associations between the HADS scores and OSDI were then assessed (figure 1A). The combination of the two components of the HADS identified that $14 \%(\mathrm{~N}=27)$ of patients had both anxiety and depression concurrently, with 3\% (N=6) having only anxiety, $7 \%(\mathrm{~N}=13)$ had only depression and the remainder $(77 \%, \mathrm{~N}=154)$ having neither. OSDI scores were found to differ significantly across these groups $(\mathrm{p}<0.001)$. On post hoc analysis, no significant difference in OSDI scores was detected between those patients with neither depression nor anxiety, and those with depression alone (median 29 vs $29, p=1.000)$. OSDI scores were not significantly raised in those with anxiety alone (median: $52, p=0.143$ ) but were found to be significantly higher in the patients with concurrent anxiety and depression (median: 72, $\mathrm{p}<0.001)$.

Associations between eye drop usage and both anxiety and depression were then assessed (figure 1B,C). On univariable analysis, anxiety scores were found to increase significantly with increasing eye drop frequency $(p<0.001)$, with rates of anxiety increasing from $4 \%$ in those that did not require eye drops, to $24 \%$ in those with an up to 2 hourly frequency. A similar trend was observed for depression $(\mathrm{p}=0.008)$, with rates increasing with frequency from $11 \%$ to $27 \%$. Subgroup analysis by disease group found similar results for OSD, although the association between eye drop frequency and depression in Uveitis did not reach statistical significance $(p=0.279)$. The association between oral glucocorticoid usage and anxiety and depression were also assessed. The total group of $\mathrm{N}=44$ (22\%) of patients taking oral glucocorticoids had an over twofold increase in both the rates of anxiety (32\% vs $12 \%, \mathrm{p}=0.005)$ and depression $(36 \%$ vs $15 \%, \mathrm{p}=0.005)$, compared with the remainder of the cohort.

Multivariable analysis was then performed, to assess whether eye drop frequencies were independently associated with the depression and anxiety scores, after accounting for other potentially confounding factors (table 3).

The depression score was found to increase significantly with the OSDI $(p<0.001)$, and to be significantly higher in the group of students and unemployed patients than those who were in employment $(p=0.007)$. After 

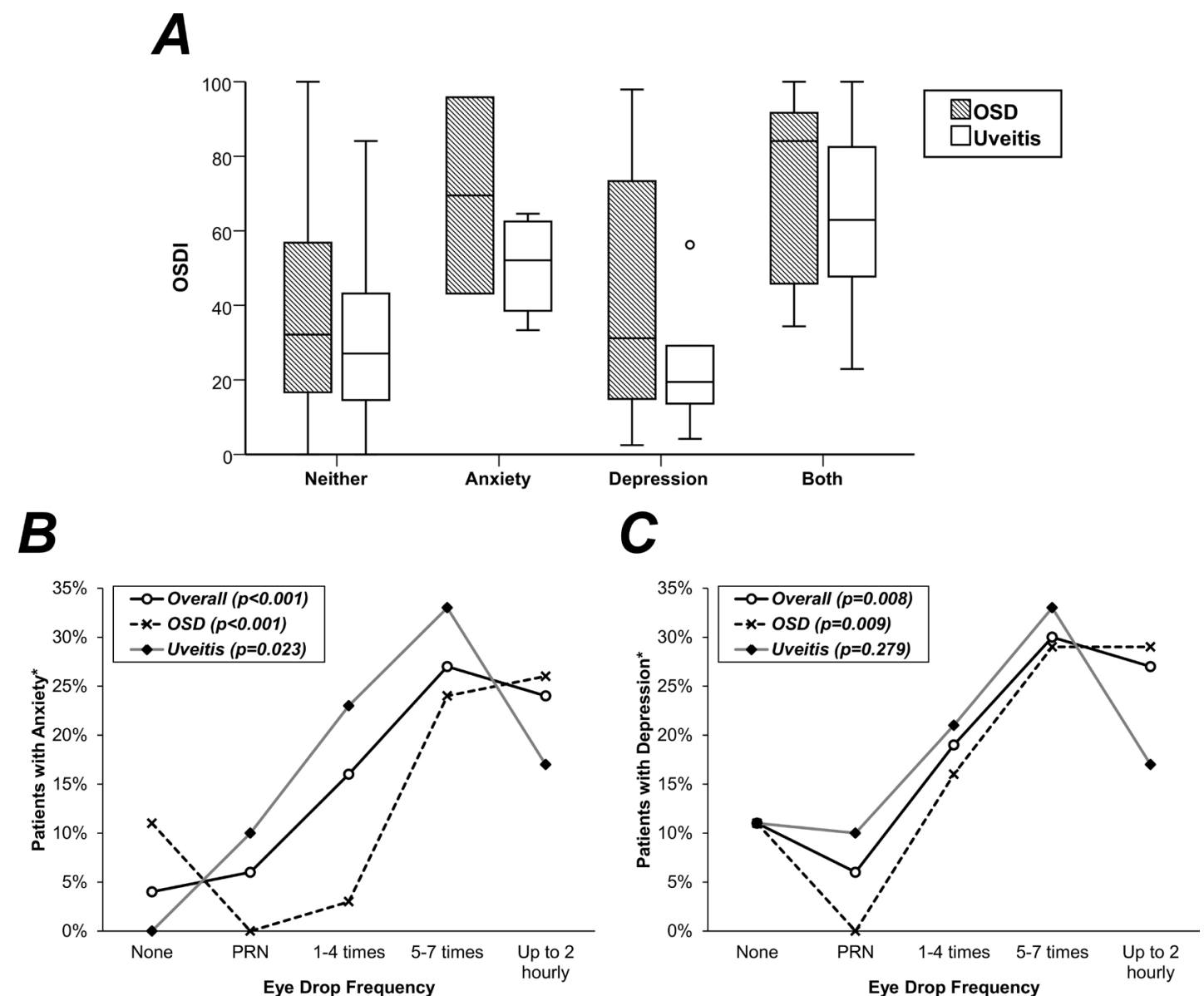

Figure 1 (A) Prevalence of ocular symptoms stratified according to anxiety and depression in patients with IED. (B) Rates of anxiety in subgroups and total cohort by frequency of eye drops. (C) Rates of depression in subgroups and total cohort by frequency of eye drops. In (A), the OSDI was found to differ significantly between the four anxiety/depression categories (Kruskal-Wallis test: $p<0.001$ ). Post hoc analysis found that the patients with both anxiety and depression had significantly higher OSDI scores than those with neither $(p<0.001)$, or with depression alone $(p=0.003)$. *Anxiety/depression is defined as HADS $\geq 11$. OSD, ocular surface disease; HADS, Hospital Anxiety and Depression Scale: PRN, as required.

accounting for these factors, the association between eye drop frequency and the depression score became nonsignificant $(\mathrm{p}=0.300)$.

The anxiety score was also found to be significantly associated with the OSDI $(\mathrm{p}<0.001)$. However, after adjusting for this, the association with eye drop frequency remained significant $(\mathrm{p}=0.009)$. Relative to patients who did not use eye drops or used them on PRN basis, patients with a frequency of 1-4 drops per day had a $36 \%(95 \%$ CI $5 \%$ to $77 \%, \mathrm{p}=0.022$ ) higher anxiety score, while those with an eye drop frequency of 5 or more per day had a $57 \%$ (95\% CI $17 \%$ to $110 \%, \mathrm{p}=0.003$ ) higher anxiety score.

Despite significance on univariable analysis, oral glucocorticoid usage was not found to be an independent predictor of either anxiety or depression in the multivariable analysis, largely since it was correlated with the OSDI.

Patients were also asked whether they become low (in mood) about the number of eye drops they are using, and the response to this question was also significantly correlated with both anxiety and depression scores (both $\mathrm{p}<0.001$, figure 2). The impact of anxiety and depression on WHOQOL-BREF scores was also assessed (online supplemental table 1). The WHOQOL-BREF scores recorded on all four domains were found to be significantly lower in those patients with HADS scores compatible with either anxiety or depression $(p<0.001$ for all comparisons).

\section{DISCUSSION}

High rates of anxiety and depression were observed in a real-world, cross-sectional analysis of the IED patient group as a whole, as measured by the HADS, with $17 \%$ being estimated to have anxiety and $20 \%$ to have depression and rates being similar in the OSD and Uveitis subgroups. These rates of anxiety and depression appear to be high, supporting previous data that there is a high prevalence of anxiety and depression in IED sufferers. ${ }^{12} 243233$ OSDI scores ranged from normal to severe and were also similar in the two disease subgroups. Those patients reporting concurrent anxiety and depression were found to have significantly worse OSDI scores and a lower QoL for all measured domains (physical, 
Table 3 Multivariable analysis of depression and anxiety scores

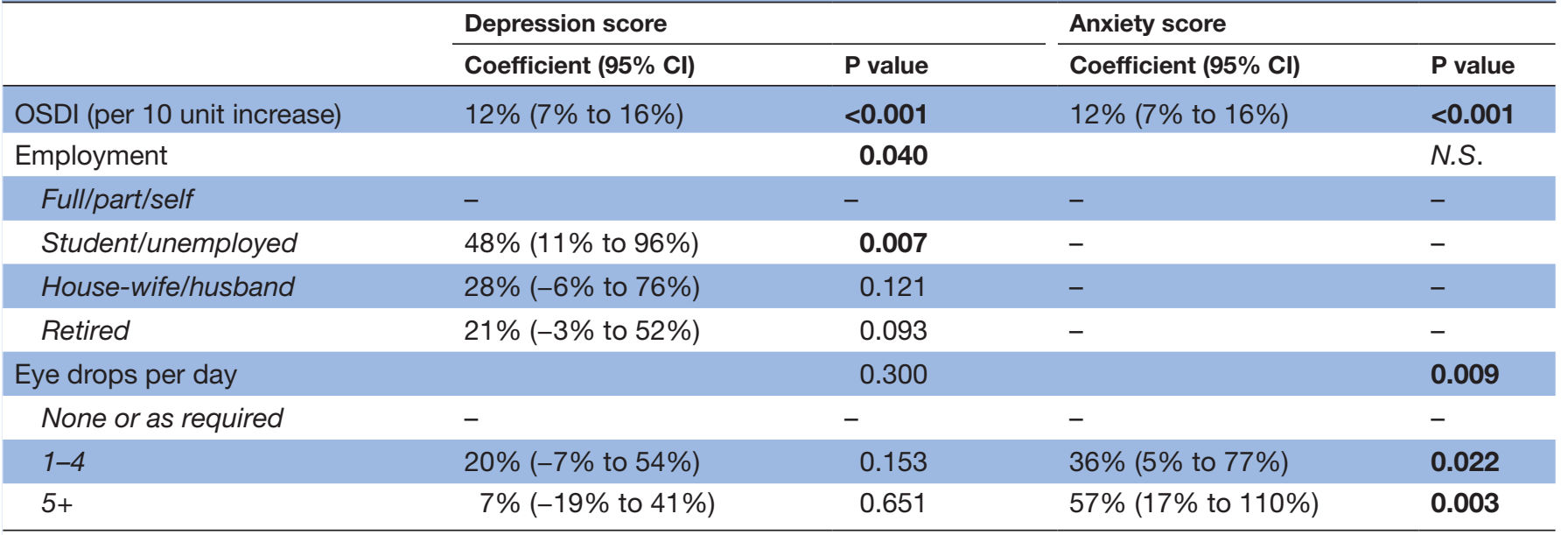

Results are from general linear models, with the log-transformed depression/anxiety scores as the dependent variables. A backward stepwise approach was used to select variables, with the following considered for inclusion: age, gender, ethnicity, education, employment, disease subgroup (OSD/Uveitis), OSDI, steroid use and eye drop frequency. The coefficients were then anti-logged and represent the percentage increase in the anxiety/depression score for the stated category relative to the reference, or for a 10-unit increase in OSDI. Bold $p$ values are significant at $p<0.05$. N.S.=not selected by the stepwise procedure for inclusion in the final model.

OSD, ocular surface disease.

psychological, social relationships, environment). The intensity of eye drop treatment was significantly associated with greater anxiety and depression and impacted on psychological status, with those reporting that they became low about the number/frequency of eye drops having significantly higher anxiety and depression scores. On multivariable analysis, OSDI was found to be the strongest predictor of anxiety and depression scores. After accounting for the OSDI score and other potentially confounding variables (including age, gender, ethnicity, education, employment, disease subgroup and corticosteroid use), the association between eye drop frequency and depression became nonsignificant. However, the association between eye drop frequency and anxiety remained significant, with the HADS anxiety score being $57 \%$ higher, on average, in those with the highest versus lowest eye drop frequencies.

In DED, there is a documented discordance between ophthalmic signs and symptoms, often with a wide variability of symptoms, which do not always correlate well with clinical signs and objective measurements, such as Schirmer's test. ${ }^{34-36}$ Instead, it has been demonstrated that DED symptomatology correlates more closely with depression than clinical signs. ${ }^{18} 3738$ This raises the question of cause and effect, and whether those with anxiety/ depression are more likely to report greater dry eye symptoms as a result of somatisation. ${ }^{33}$ Patients in our study whose HADS scores were compatible with either

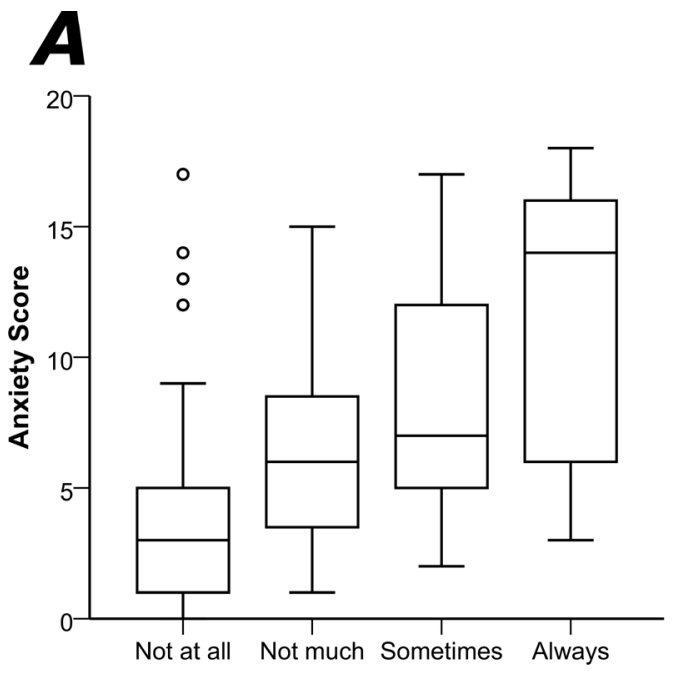

Get low about the number of drops

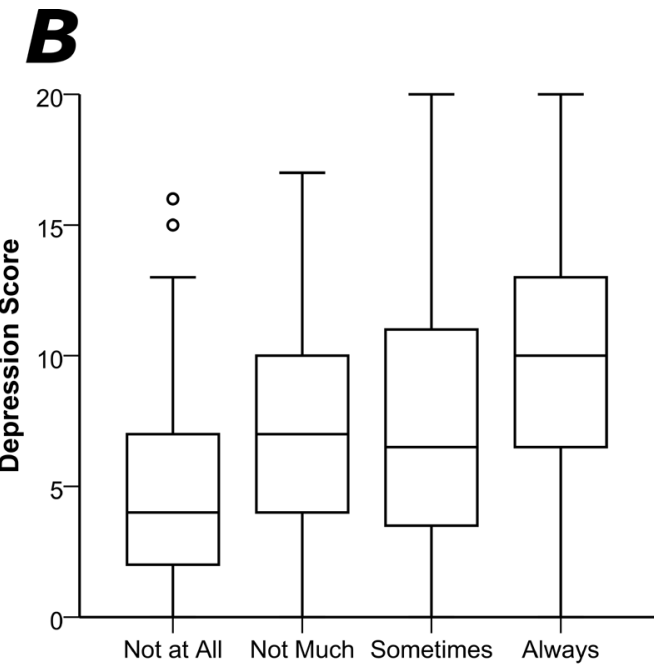

Get low about the number of drops

Figure 2 The influence of the frequency of drops on the (A) anxiety and (B) depression components of the HADS score. Patients who reported that they became 'low' in mood about the number of eye drops they used had significantly higher anxiety (Kendall's $\tau: p<0.001)$ and depression $(p<0.001)$ scores. HADS, Hospital Anxiety and Depression Scale. 
depression or anxiety alone did not report significantly worse ocular symptomatology than our nondepressed patients, as measured by the OSDI, while those with concurrent depression and anxiety did. Interestingly, our study found anxiety to increase significantly with drop frequency, even after accounting for the effect of the OSDI score, although the same was not true for depression. Unravelling the complex relationship between ocular disease severity and psychological status is no simple task, although our data suggest that the frequency of topical treatment potentially plays a significant role, at least where patient levels of anxiety are concerned.

Other variables known to be of significance in mental illness in the context of IED include systemic medical conditions, particularly chronic pain syndromes and psychiatric history, including use of antidepressants. ${ }^{75} 39$ It is known that having a chronic physical illness increases the likelihood of anxiety/depression, and that functional outcomes of physical illnesses are also worse in context of depression. It is likely that the interplay between IED, chronic pain syndromes and anxiety/depression is such that these conditions exacerbate one another, highlighting the importance of recognising mental health disorders in the context of chronic physical health conditions and vice versa. The present study did not consider patient psychiatric history, nor systemic medical conditions. Further research on the role of topical eye treatment frequency might additionally consider these variables with added support from a psychiatrist/psychologist for diagnostic confirmation of prioror current clinical anxiety and depression. Psychologist input would also be valuable in performing qualitative reviews to further support concept elicitation presented here, for cognitive interviews that support content validity and subsequent quantitative verification. However, this was outside of the scope of the current study.

We believe that our study addresses a topic of significant public health importance and highlights the psychological problems associated with the use of frequent eye drops. Although the fact that medical treatments can have psychological implications is not a new finding, the medical community is currently failing to reach the high standards required to mitigate this, thus the significance of this research. ${ }^{4041}$ Additionally, we engaged with patients from two PPI groups, who positively influenced our methodology. Furthermore, by using OSDI in both patient groups, we indicated the potential use of OSDI with uveitis, and we provided a better understanding about how uveitis influences the activities of daily living. Finally, this is the first study investigating, using realworld data, the influence of eye drops on a patient's well-being and can be used as a starting point for further assessment of the health status and care required for our patients. All these are important findings and considerably strengthen our study.

However, our study has some limitations. This is a cross-sectional study and may be subject to bias in relation to whether the anxiety and depression preceded, or resulted from, the use of eye drops. In addition, as the patients are attending specialised tertiary referral clinics, they are likely to be those with more severe disease that may be chronic in nature and require extensive therapy. As such, the observed effects on psychological status may not be generalisable to patients with milder forms of disease. Finally, we did not collect data on the types of eye drop used (corticosteroid vs mydriatics vs topical immune suppressants vs ocular lubricants) and so were not able to assess whether associations with psychological status varied with the specific ocular medication used. However, our aim was to assess the influence that the eye drops have on our patients' psychology in general, rather the effects of individual formulations.

Despite research suggesting that treatments for mental health conditions in context of chronic illness may improve both mental and physical health outcomes, current practice still largely continues not to integrate treatments accordingly. ${ }^{4}{ }^{4}$ If further research were to validate the impact of treatment burden (as measured by frequency of eye drops) on patients with IED and their well-being, it may support the integration of a clinical psychology service working in association with IED clinics (similar to that undertaken in the Centres of Excellence for Behçet's Syndrome in England) ${ }^{43}$ or in an eye clinic support worker (eye clinic liaison officer) with a specific interest in resolving treatment-related emotional stress.

In conclusion, in our real-world cohort, a large proportion of our IED cohort demonstrated HADS scores compatible with anxiety and depression, with associated poor QoL, in keeping with previous studies. No significant differences in patient demographics or QoL scores were found when comparing OSD and Uveitis subgroups, suggesting a comparable psychological status in these IED groups. In this study, the frequency of topical eye drops was found to be a significant independent predictor of patient anxiety scores. Together with further supporting evidence, this may contribute to the development of a more integrated and holistic approach to IED care involving psychological, emotional and practical support, which could help to achieve better clinical outcomes for our patients.

Contributors All authors fulfil the authorship criteria for this paper/research.

Funding Dr Rauz is Principal Investigator on the NIHR product development award: NIHR i4i II-LA-1117-20001 "Fluid-Gels as Resorbable Protective Dressings for Ocular Surface Disease"

Competing interests None declared.

Patient consent for publication Not required.

Provenance and peer review Not commissioned; externally peer reviewed.

Data availability statement Data are available upon request;

Supplemental material This content has been supplied by the author(s). It has not been vetted by BMJ Publishing Group Limited (BMJ) and may not have been peer-reviewed. Any opinions or recommendations discussed are solely those of the author(s) and are not endorsed by BMJ. BMJ disclaims all liability and responsibility arising from any reliance placed on the content. Where the content includes any translated material, BMJ does not warrant the accuracy and reliability of the translations (including but not limited to local regulations, clinical guidelines, terminology, drug names and drug dosages), and is not responsible 
for any error and/or omissions arising from translation and adaptation or otherwise.

Open access This is an open access article distributed in accordance with the Creative Commons Attribution Non Commercial (CC BY-NC 4.0) license, which permits others to distribute, remix, adapt, build upon this work non-commercially, and license their derivative works on different terms, provided the original work is properly cited, appropriate credit is given, any changes made indicated, and the use is non-commercial. See: http://creativecommons.org/licenses/by-nc/4.0/.

ORCID iDs

Georgios Vakros http://orcid.org/0000-0002-5673-2003

Philip I Murray http://orcid.org/0000-0001-8491-3795

\section{REFERENCES}

1 American Psychiatric Association. Diagnostic and statistical mental disorders (DSM 5). American Psychiatric Association, 2013: 947. https://dsm.psychiatryonline.org/doi/full/

2 Zajecka JM, Ross JS. Management of comorbid anxiety and depression. J Clin Psychiatry 1995;56 Suppl 2:10-13.

3 Appleby L, Asherson P, Aznar C. Adult psychiatric morbidity survey 2014. Ment Heal Wellbeing Engl 2014.

4 Clarke DM, Currie KC. Depression, anxiety and their relationship with chronic diseases: a review of the epidemiology, risk and treatment evidence. Med J Aust 2009;190:S54-60.

5 Evans DL, Charney DS, Lewis L, et al. Mood disorders in the medically ill: scientific review and recommendations. Biol Psychiatry 2005;58:175-89.

6 Li M, Gong L, Chapin WJ, et al. Assessment of vision-related quality of life in dry eye patients. Invest Ophthalmol Vis Sci 2012;53:5722-7.

7 Qian Y, Glaser T, Esterberg E, et al. Depression and visual functioning in patients with ocular inflammatory disease. Am J Ophthalmol 2012;153:370-8.

8 Paulsen AJ, Cruickshanks KJ, Fischer ME, et al. Dry eye in the Beaver dam offspring study: prevalence, risk factors, and healthrelated quality of life. Am J Ophthalmol 2014;157:799-806.

9 Hallak JA, Tibrewal S, Jain S. Depressive symptoms in patients with dry eye disease: a case-control study using the Beck depression inventory. Cornea 2015;34:1545-50.

10 Yilmaz U, Gökler ME, Unsal A. Dry eye disease and depressionanxiety-stress: a hospital-based case control study in turkey. Pak J Med Sci 2015;31:626-31.

11 Zhang $\mathrm{Y}$, Lin T, Jiang A, et al. Vision-related quality of life and psychological status in Chinese women with Sjogren's syndrome dry eye: a case-control study. BMC Womens Health 2016;16:75.

12 Stack RJ, Southworth S, Fisher BA, et al. A qualitative exploration of physical, mental and ocular fatigue in patients with primary Sjögren's Syndrome. PLoS One 2017;12:e0187272.

13 Liyue H, Chiang PP-C, Sung SC, et al. Dry Eye-Related visual Blurring and irritative symptoms and their association with depression and anxiety in eye clinic patients. Curr Eye Res 2016;41:590-9.

14 Hoeksema L, Los LI. Vision-related quality of life in herpetic anterior uveitis patients. PLoS One 2014;9:e85224.

15 Hui MM, Wakefield D, Patel I, et al. Visual functioning and healthrelated quality-of-life are compromised in patients with uveitis. Ocul Immunol Inflamm 2017;25:486-91.

16 Hoeksema L, Los LI. Vision-related quality of life in patients with inactive HLA-B27-associated-spectrum anterior uveitis. PLoS One 2016;11:e0146956.

17 Schiesser A, Katzenschlager S, Benetka G. Multimodel treatment of chronified, non-malignant pain and its influence on pain behavior. In: Hu F, ed. Pain research progress: migraine, fibromyalgia and related pain. Hauppauge, NY: Nove Science Publishers, 2008: 151-74.

18 Galor A, Felix ER, Feuer W, et al. Dry eye symptoms align more closely to non-ocular conditions than to tear film parameters. $\mathrm{Br} J$ Ophthalmol 2015;99:1126-9.
19 Galor A, Zlotcavitch L, Walter SD, et al. Dry eye symptom severity and persistence are associated with symptoms of neuropathic pain. Br J Ophthalmol 2015;99:665-8.

20 Galor A, Moein H-R, Lee C, et al. Neuropathic pain and dry eye. Ocul Surf 2018;16:31-44.

21 Wolffsohn JS, Arita R, Chalmers R, et al. TFOS DEWS II diagnostic methodology report. Ocul Surf 2017;15:539-74.

22 Crane AM, Levitt RC, Felix ER, et al. Patients with more severe symptoms of neuropathic ocular pain report more frequent and severe chronic overlapping pain conditions and psychiatric disease. Br J Ophthalmol 2017;101:227-31.

23 Javidi H, Poonit N, Patel RP, et al. Adherence to topical medication in patients with inflammatory eye disease. Ocul Immunol Inflamm 2020:1-6

24 Maca SM, Schiesser AW, Sobala A, et al. Distress, depression and coping in HLA-B27-associated anterior uveitis with focus on gender differences. Br J Ophthalmol 2011;95:699-704.

25 Maca SM, Wagner J, Weingessel B, et al. Acute anterior uveitis is associated with depression and reduction of general health. $\mathrm{Br} J$ Ophthalmol 2013;97:333-7.

26 Schiffman RM, Christianson MD, Jacobsen G, et al. Reliability and validity of the ocular surface disease index. Arch Ophthalmol 2000;118:615-21.

27 Karaca I, Palamar M, Guven Yilmaz S, et al. Evaluation of ocular surface and meibomian glands alterations with Meibography in patients with inactive Behcet's uveitis. Curr Eye Res 2019;44:356-9.

28 Zigmond AS, Snaith RP. The hospital anxiety and depression scale. Acta Psychiatr Scand 1983;67:361-70.

29 Brennan C, Worrall-Davies A, McMillan D, et al. The hospital anxiety and depression scale: a diagnostic meta-analysis of case-finding ability. J Psychosom Res 2010;69:371-8.

30 Bjelland I, Dahl AA, Haug TT, et al. The validity of the hospital anxiety and depression scale. An updated literature review. J Psychosom Res 2002;52:69-77.

31 Skevington SM, Lotfy M, O'Connell KA, et al. The world Health organization's WHOQOL-BREF quality of life assessment: psychometric properties and results of the International field trial. A report from the WHOQOL group. Qual Life Res 2004;13:299-310.

32 Li M, Gong L, Sun X, et al. Anxiety and depression in patients with dry eye syndrome. Curr Eye Res 2011;36:1-7.

33 Wan KH, Chen LJ, Young AL. Depression and anxiety in dry eye disease: a systematic review and meta-analysis. Eye 2016;30:1558-67.

34 Craig JP, Nelson JD, Azar DT, et al. TFOS DEWS II report executive summary. Ocul Surf 2017;15:802-12.

35 Vehof J, Sillevis Smitt-Kamminga N, Nibourg SA, et al. Predictors of discordance between symptoms and signs in dry eye disease. Ophthalmology 2017;124:280-6.

36 Buckley RJ. Assessment and management of dry eye disease. Eye 2018;32:200-3.

37 Labbé A, Wang YX, Jie Y, et al. Dry eye disease, dry eye symptoms and depression: the Beijing eye study. Br J Ophthalmol 2013;97:1399-403.

38 Szakáts I, Sebestyén M, Németh J, et al. The role of health anxiety and depressive symptoms in dry eye disease. Curr Eye Res 2016:41:1044-9.

39 Vehof J, Sillevis Smitt-Kamminga N, Kozareva D, et al. Clinical characteristics of dry eye patients with chronic pain syndromes. Am $J$ Ophthalmol 2016;162:59-65.

40 Jeffrey D, Empathy JD. Empathy, sympathy and compassion in healthcare: is there a problem? is there a difference? does it matter? $J$ R Soc Med 2016;109:446-52.

41 Fellow-Smith E, Moss-Morris R, Tylee A. Investing in emotional and psychological wellbeing for patients with long-term conditions, 2012 Available: https://www.nhsconfed.org/-/media/Confederation/Files/ Publications/Documents/Investing-in-emotional-and-psychologicalwellbeing-for-patients-with-long-term-condtions-16-April-final-forwebsite.pdf [Accessed Dec 2020]

42 Wulsin LR, Vaillant GE, Wells VE. A systematic review of the mortality of depression. Psychosom Med 1999;61:6-17.

43 Moots RJ, Fortune F, Situnayake D. The Behçet's centres of excellence. Rheumatology 2018;57:594-5. 